\title{
Robo-Fish Replicates Real Swimming Action
}

\author{
A robotic fish whose swimming action is initiated in the same way as that \\ of real fish could help researchers test predictions about these \\ underwater creatures, using well-controlled conditions.
}

By Katherine Wright

A long with the five senses of touch, sight, hearing, smell, and taste, fish can sense deformations of their bodies that link to their position in the water and the speed at which they swim. Researchers think that this ability, which is known as proprioception, or, colloquially, as the "sixth sense," is used by fish to orient their fins for optimal swimming. Now a team of researchers has demonstrated a robot fish whose tail movements are controlled by proprioception. They say that the robo-fish could be used to study idealized swimming actions that are impossible to observe in living things.

The robot, which was designed and built by Médéric Argentina of the Côte d'Azur University, France, and his colleagues, resembles the skeleton of a fish. The head, backbone, scales, and tail (the robot has no fins) were separately 3D printed from various elastic materials and then glued together. A servomotor was attached to the tail using two cables, which drive side-to-side motion of the tail using information collected by a

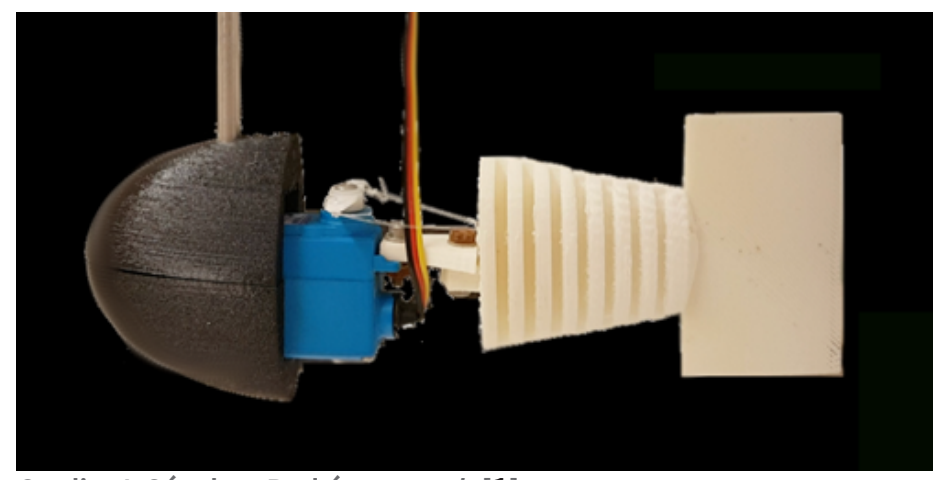

Credit: J. Sánchez-Rodríguez et al. [1]

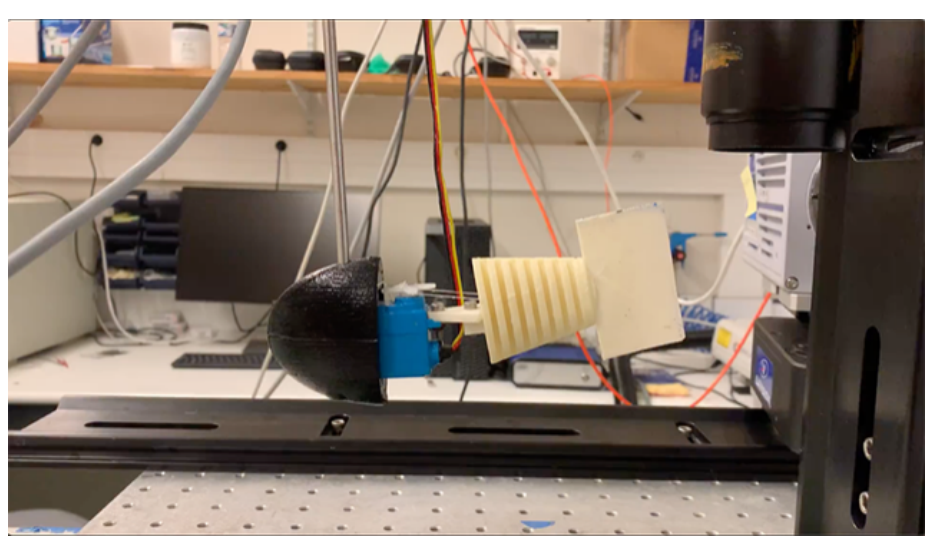

Video 1: Researchers in France replicate the sense of proprioception in a robot fish by linking the action of a motor that drives movements of the robot's tail to force sensors on its head. Credit: J. Sánchez-Rodríguez et al. [1]

force sensor on the robot's head.

The team observed that this force sensing induces the body of the fish to undulate and the tail to move from side to side in a manner that matches that of real fish. This motion only occurs when the robot is submerged and stops when the robot emerges above the waterline. Argentina says that the robot "chooses" when and how to swim: the motion is completely determined by a feedback loop between the motor and the force sensor and is not programmed into the robot. That ability to determine its own motion makes this design interesting for use in future autonomous vehicles, the team says.

Katherine Wright is the Deputy Editor of Physics. 
REFERENCES

1. J. Sánchez-Rodríguez et al., "Proprioceptive mechanism for bioinspired fish swimming," Phys. Rev. Lett. 126, 234501 (2021). 